\title{
A Heuristic Multiobjective Method for Radial Distribution Networks Reconfiguration
}

\author{
Shahrokh Shojaeian and Ebrahim Ghandehari \\ Department of Electrical Engineering, Khomeinishahr Branch, Islamic Azad University, P.O. Box 84175-119, Isfahan 8418148499, Iran \\ Correspondence should be addressed to Shahrokh Shojaeian; shojaeian@iaukhsh.ac.ir
}

Received 20 August 2013; Accepted 7 October 2013

Academic Editors: I. Smith and B. Suman

Copyright ( 2013 S. Shojaeian and E. Ghandehari. This is an open access article distributed under the Creative Commons Attribution License, which permits unrestricted use, distribution, and reproduction in any medium, provided the original work is properly cited.

\begin{abstract}
This paper introduces a novel algorithm for radial distribution networks reconfiguration, called "Sifting algorithm." It not only has a simple structure but also has high speed and accuracy. It works by eliminating infeasible states and reduces the search space and then it uses a simple method to find optimum answer in remaining space. To ensure the effectiveness of this algorithm, a 16-switch test network was tested and the results were compared with three other well-known algorithms. The results of simulations showed high speed and accuracy of this algorithm.
\end{abstract}

\section{Introduction}

Due to daily growth of electrical energy consumers and available limitations of generation and transmission of energy, distribution systems are often operating under overload conditions. The statistical results showed that majority of interruptions of energy come from distribution systems. For this reason these systems are taken in consideration recently. Some of the most important objectives for optimization include reducing, total losses of system (due to economic attractiveness), improving reliability of system (due to reduce faults) and improving voltage profile (due to consumers satisfaction). Optimization of these systems is done by a usual method that is called "reconfiguration" or "restructuring." Reconfiguration will change structure of the network by changing status of its switches. These switches can be divided into two categories including sectionalizing (normally close) switches and tie (normally open) switches [1]. In fact for changing structure of the network, the switches states should be changed. Since the most distribution networks all over the world are radial, there are some specific limitations for them. Some of these limitations include maintaining radial structure, establishing load balancing, not overloading equipment and, and so forth [2]. The other work of reconfiguration is introduction of switching scenarios of the network. It will help operators to take the best decision in a minimum time. It minimizes human faults and does not need to shut down the system too [3].

The most important data for reconfiguration include load consummation patterns and data of system. Usually, it is assumed that loads are constant, but sometimes they are taken into consideration as probabilistic. To obtain this information such systems like Customer Information Systems (CIS) and Outage Management Information System (OMIS) can be used [4]. Reconfiguration can be done automatically in the smart networks $(3 \mathrm{G})$. In these networks the status of system is observable online and switches can be controlled remotely [5]. Network reconfiguration is useful when a fault occurs in system too. At the time, fault point will isolate and structure of network will change. Usually it is assumed that fault point will be found as soon as possible [6].

Changing the status of the switches can be done by a lot of algorithms, but since power system problems are combinatorial optimization, they are difficult to solve by traditional linear or nonlinear methods; then optimization algorithms should be used [3]. Some of these optimization algorithms include genetic algorithm (GA), ant colony search algorithm (ACSA), artificial bee colony algorithm (ABC), simulated annealing (SA), Tabu search, particle swarm optimization (PSO), heuristic algorithms, and so forth [7-12]. The present 


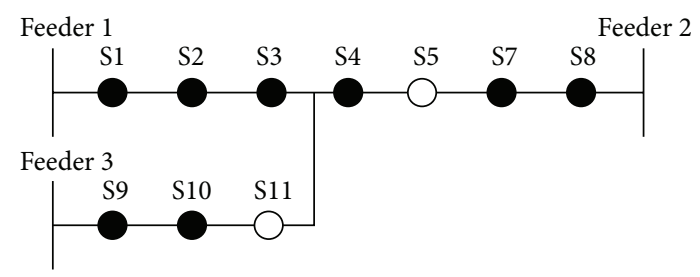

Figure 1: A typical network to explain binary code generation.

paper will introduce a novel algorithm that is an invention of authors and not only has high speed, but also its accuracy and convergence are very good.

One more important issue in the reconfiguration problem is objective function. The most important objective in the recent literature is loss minimization. But there are more objectives which can be considered, too, such as reliability, voltage deviation, load balancing, transient behaviors of the network, smart grid interactions, and so forth [6,12-14]. This paper optimizes two objectives including voltage deviation and total system losses.

\section{The Reconfiguration Process}

The first step to solve reconfiguration problem is representation of the network structure with a comprehensive code for computer. To show the status of $N$ switches in a distribution feeder, a string of $N$ bit can be used. For each switch, "1" shows that its status is closed and "0" shows it is open. For example, in the feeders shown in Figure 1 the above mentioned code is (11110111110).

If the statuses of switches change, their string code will change too. Then reconfiguration problem is a binary issue, because all states of the network are combinations of 0 and 1. An optimum code for the network has to be found which results in minimum value of the objective function. For a distribution network with $N$ switches there are $2^{N}$ possible codes which all of them are not necessarily feasible.

One advantage of the reconfiguration problem is its unfeasible solutions that are predictable. Then search space can be reduced. There are different ways to determine feasible states. Three ways of them will be introduced in the following that everyone has advantages and disadvantages.

The First Method. Generate a random binary code and restructure the network by it. If all buses are fed just from one side, this code is feasible and should be saved; otherwise, it is not. The speed of this method is low.

The Second Method. Figure 2 explains this method. Here the network has three feeders and it is obvious that there must not be any closed loop between them, and all buses must be fed too. So all connection ways between feeders should be found and every network code must have just one 0 . If there are more, that code is infeasible. The other important limitation is that switches of end loads or feeders should be 1. To understand this method, see Figure 2. Connection ways between feeders include down branch (codes 13, 14,

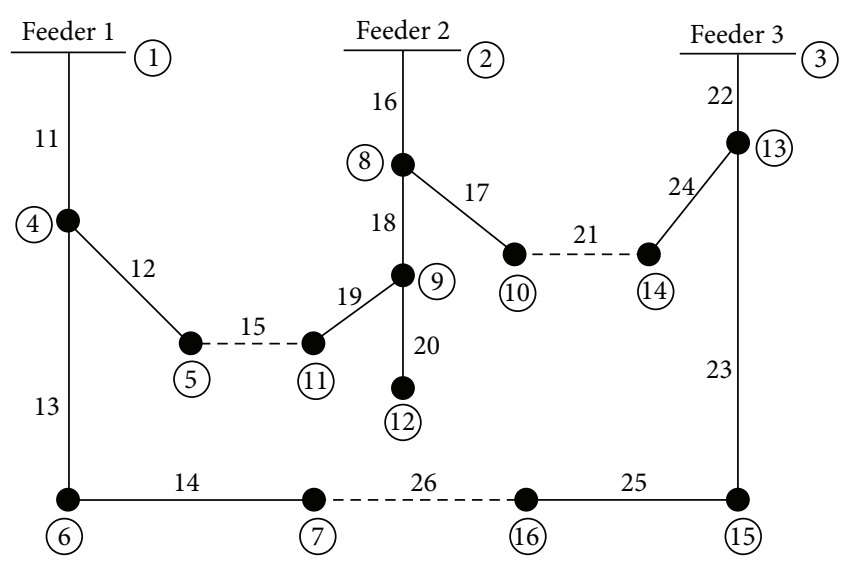

FIGURE 2: 3-feeder distribution network.

26, 25, and 23), left branch (codes 12,15,19, and 18), and right branch (codes 17, 21 and 24). In every three codes, there should be just one 0 to meet network structure feasibility. All feasible states of the network are equal to multiplication of the number of all collected codes. For example in Figure 2 the number of feasible codes is 60 ; this number is gotten from the multiplication of five down branch codes by four left branch codes by three right branch codes. This method is harder but it is very fast and reliable. Flowchart of Figure 3 will explain this method.

The Third Method. The desired codes can be generated manually, for example, by Microsoft Office Excel and then saved in a database. This way is not automatic but is very fast.

\section{Sifting Algorithm}

As mentioned above, infeasible answers of reconfiguration problem are predictable. Then search space can be reduced. Sifting method is a heuristic algorithm which does this reduction very well.

The sifting algorithm is almost similar to the well-known PSO algorithm, with a few differences in generation first code and swarm position update. In the proposed method at the first step one of the feasible codes is selected and network reconfiguration is done. This process is repeated in the times equal to the population number. Then, one answer is selected as the best answer (BA). At the next step, some codes which are differed at just one switch (or bit) by BA are selected. Indeed these codes are most similar codes to BA and they can be optimum answer. Then each of them is selected and verified. If each of them was better than $B A$, that code will be the new BA. This process will continue until convergence is met. Flowchart of Figure 4 will explain sifting algorithm.

The advantages of all these methods over the other methods are their high speed of convergence and simple structure. This method is fast because generated codes are feasible, then after a few repetitions a good answer (BA) can be found. At the next step, the most similar states to BA will be selected and verified; these states are different at just one or two switches. It avoids the algorithm to trap 


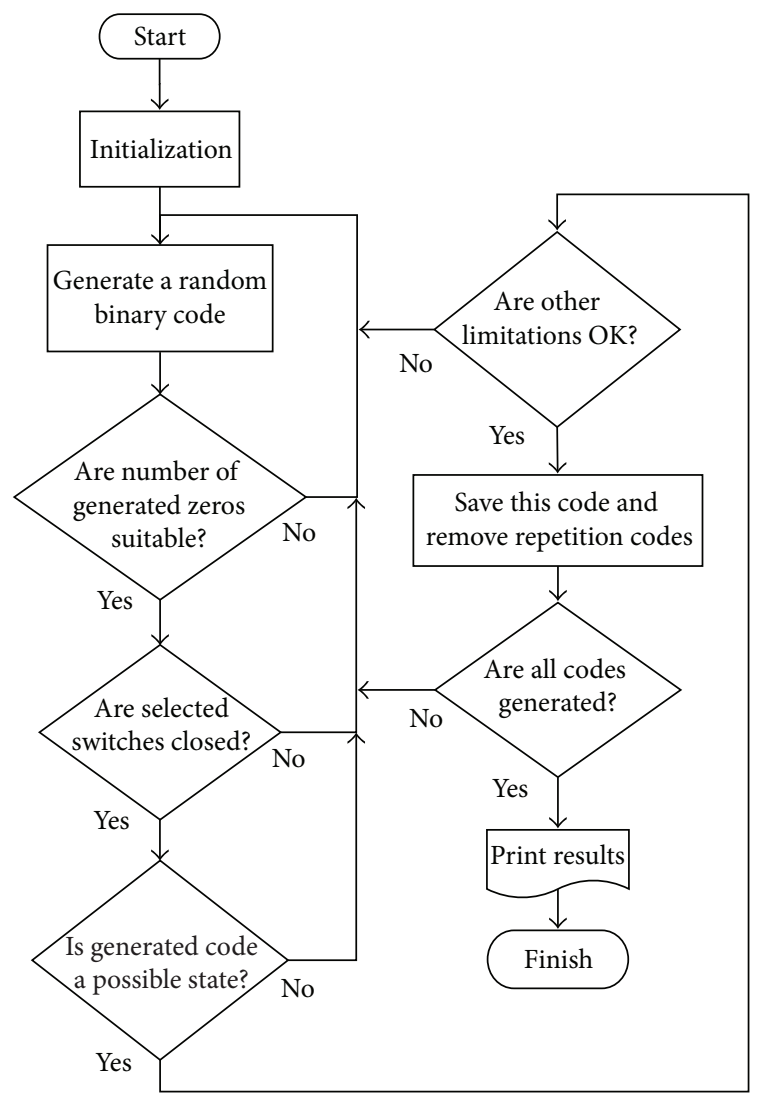

FIGURE 3: Flowchart of random codes making method.

in a local optimum solution. At this step just feasible codes will be checked, but in other algorithms like BPSO codes will be updated and then changed to feasible states $[15,16]$. This change will reduce the speed of algorithm and make it difficult too.

One more advantage of the proposed method is that it guarantees fast convergence because answer space will reduce significantly. For example, in Figure 2 feasible states are equal to 60 (as already mentioned), but all states are equal to $2^{16}$ $(=65536)$; then answer space is about $0.09 \%$ of the search space.

\section{Simulation and Results}

For a well-known distribution network shown in Figure 2, which has been used in many literatures before, the proposed method is applied. The network parameters can be found in [17]. To show the speed and the effectiveness of the proposed method, a comparison is made between this method and three other algorithms. Table 1 shows reconfiguration CPU times to minimize total losses and voltage deviation of the network by four different methods. It is clear that sifting method's time is much less than the others (more than 4 times) and if the number of the network bus increases, the CPU time difference will be obviously more. The final solution is the same in all algorithms.

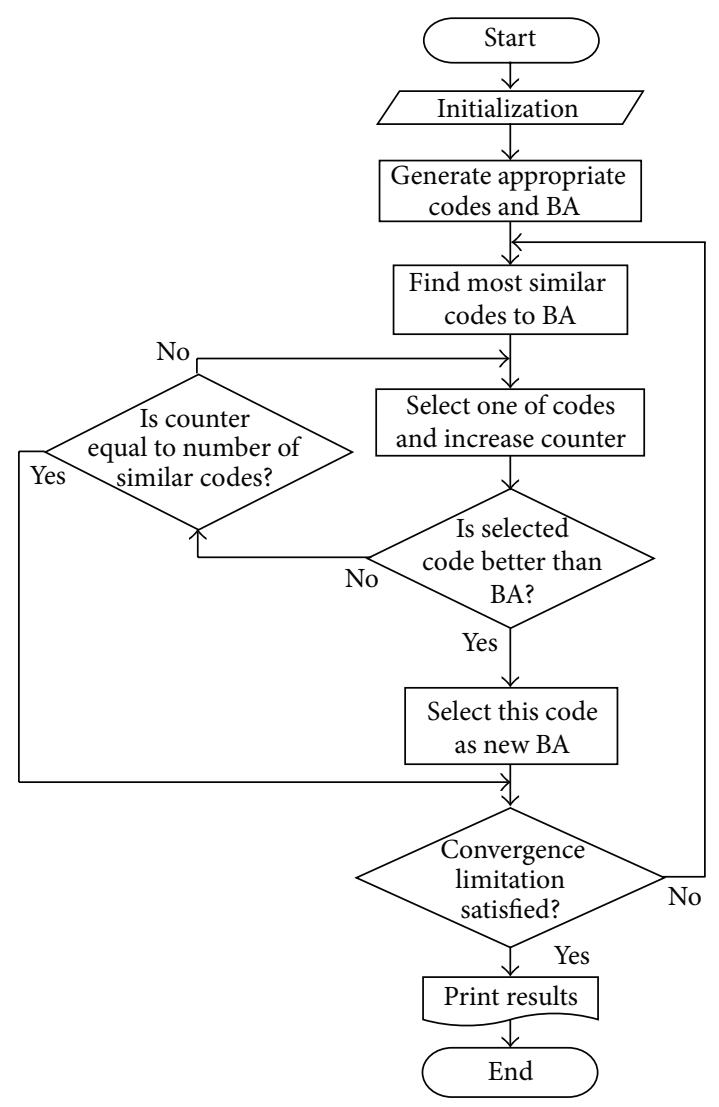

FIGURE 4: Flowchart of the sifting algorithm.

TABLE 1: Obtained results for the proposed method and the methods described in [3].

\begin{tabular}{lcccc}
\hline \multirow{2}{*}{ Methods } & \multicolumn{3}{c}{ Reference [3] } & \multirow{2}{*}{ Sifting procedure } \\
& GA & SA & ACSA & \\
\hline $\begin{array}{l}\text { CPU time } \\
\text { (seconds) }\end{array}$ & 2.32 & 2.07 & 1.81 & $0.43^{*}$ \\
$\begin{array}{l}\text { Population } \\
\begin{array}{l}\text { Best position } \\
\text { (Open switches) }\end{array}\end{array}$ & 5 & 500 & 5 & 8 \\
\hline
\end{tabular}

${ }^{*}$ This time is average of 10 runs.

The objective function (O.F.) of this problem which includes the total losses and the voltage deviation of the feeders is shown as follows:

$$
\text { O.F. }=\alpha \cdot \text { Loss }+\beta \cdot \text { Voltage deviation, }
$$

where $\alpha$ and $\beta$ are weighting coefficients which show relative importance of the components. These coefficients are obtained with historical experiences and strategies of each distribution system company. In this paper they chose 1 and 2, respectively. Total losses and cumulative voltage deviation 
after running a load flow program will be obtained by the following:

$$
\begin{gathered}
\text { Loss }=\sum_{i=0}^{N-1} r_{i} \frac{P_{i}^{2}+Q_{i}^{2}}{V_{i}^{2}} \mathrm{pu}, \\
\text { Voltage deviation }=\sum_{i=0}^{N-1}\left(V_{i}-1\right)^{2} \mathrm{pu},
\end{gathered}
$$

where $V_{i}$ is the voltage magnitude of the $i$ th bus and $P_{i}, Q_{i}$, and $r_{i}$ are the active and reactive power and resistance of the $i$ th section, respectively.

\section{Conclusion}

In this paper, a new method called "sifting algorithm" was introduced for radial distribution networks reconfiguration. This algorithm works based on eliminating infeasible states in the search space. Then a forward/backward load flow method was applied for feasible states. An objective function containing the power loss and the voltage deviation was calculated and the best configuration was recognized. To ensure the efficiency of the proposed algorithm, a standard 16-switch network was tested and the results are compared with three other well-known algorithms. The simulation results were very satisfactory, so that the speed of the proposed algorithm was about 4 times better than the best answer of the other algorithms.

\section{Acknowledgment}

The authors wish to thank the Islamic Azad University, Khomeinishahr branch, Isfahan, Iran, for its support.

\section{References}

[1] B. Amanulla, S. Chakrabarti, and S. N. Singh, "Reconfiguration of power distribution systems considering reliability and power loss," IEEE Transactions on Power Delivery, vol. 27, no. 2, pp. 918-926, 2012.

[2] M. W. Siti, D. V. Nicolae, A. A. Jimoh, and A. Ukil, "Reconfiguration and load balancing in the LV and MV distribution networks for optimal performance," IEEE Transactions on Power Delivery, vol. 22, no. 4, pp. 2534-2540, 2007.

[3] Y.-K. Wu, C.-Y. Lee, L.-C. Liu, and S.-H. Tsai, "Study of reconfiguration for the distribution system with distributed generators," IEEE Transactions on Power Delivery, vol. 25, no. 3, pp. 1678-1685, 2010.

[4] L. S. M. Guedes, A. C. Lisboa, D. A. G. Vieira, and R. R. Saldanha, "A multiobjective heuristic for reconfiguration of the electrical radial network," IEEE Transaction on Power Delivery, vol. 28, pp. 311-319, 2013.

[5] Y.-L. Ke, "Distribution feeder reconfiguration for load balancing and service restoration by using G-Nets inference mechanism," IEEE Transactions on Power Delivery, vol. 19, no. 3, pp. 1426-1433, 2004.

[6] V. Spitsa, X. Ran, R. Salcedo et al., "On the transient behavior of large-scale distribution networks during automatic feeder reconfiguration," IEEE Transaction on Smart Grid, vol. 3, pp. 887-896, 2012.
[7] H. D. De Macedo Braz and B. A. de Souza, "Distribution network reconfiguration using genetic algorithms with sequential encoding: subtractive and additive approaches," IEEE Transactions on Power Systems, vol. 26, no. 2, pp. 582-593, 2011.

[8] A. Saffar, R. Hooshmand, and A. Khodabakhshian, "A new fuzzy optimal reconfiguration of distribution systems for loss reduction and load balancing using ant colony search-based algorithm," Applied Soft Computing Journal, vol. 11, no. 5, pp. 4021-4028, 2011.

[9] J. Olamaei, T. Niknam, and S. B. Arefi, "Distribution feeder reconfiguration for loss minimization based on modified honey bee mating optimization algorithm," in Proceedings of the 2nd International Conference on Advances in Energy Engineering, pp. 304-311, December 2011.

[10] V. Parada, J. A. Ferland, M. Arias, and K. Daniels, "Optimization of electrical distribution feeders using simulated annealing," IEEE Transactions on Power Delivery, vol. 19, no. 3, pp. 1135-1141, 2004.

[11] A. Y. Abdelaziz, F. M. Mohamed, S. F. Mekhamer, and M. A. L. Badr, "Distribution system reconfiguration using a modified Tabu Search algorithm," Electric Power Systems Research, vol. 80, no. 8, pp. 943-953, 2010.

[12] H.-T. Yang, Y.-T. Tzeng, and M.-S. Tsai, "Loss-minimized distribution system reconfiguration by using improved multiagent based particle swarm optimization," in Proceedings of the Asia-Pacific Power and Energy Engineering Conference (APPEEC '10), March 2010.

[13] B. Amanulla, S. Chakrabarti, and S. N. Singh, "Reconfiguration of power distribution systems using probabilistic reliability models," in Proceedings of the IEEE PES General Meeting: The Electrification of Transportation and the Grid of the Future, July 2011.

[14] F.-Y. Hsu and M.-S. Tsai, "A multi-objective evolution programming method for feeder reconfiguration of power distribution system," in Proceedings of the 13th International Conference on Intelligent Systems Application to Power Systems (ISAP '05), pp. 55-60, November 2005.

[15] Y. Qin and J. Wang, "Distribution network reconfiguration based on particle clonal genetic algorithm," Journal of Computers, vol. 4, no. 9, pp. 813-820, 2009.

[16] M. S. Tsai and W. C. Wu, "A novel binary coding particle swarm optimization for feeder reconfiguration," in Particle Swarm Optimization, A. Lazinica, Ed., chapter 27, 2009.

[17] C.-T. Su, C.-F. Chang, and J.-P. Chiou, "Distribution network reconfiguration for loss reduction by ant colony search algorithm," Electric Power Systems Research, vol. 75, no. 2-3, pp. 190199, 2005. 

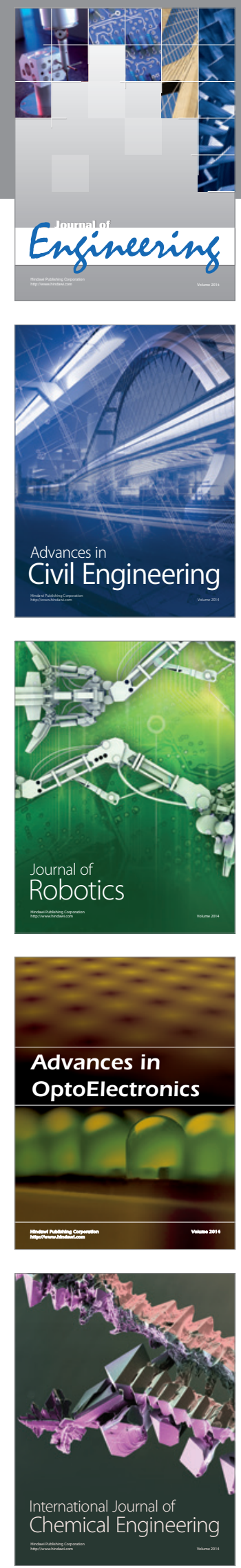

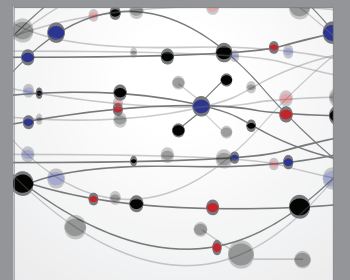

The Scientific World Journal
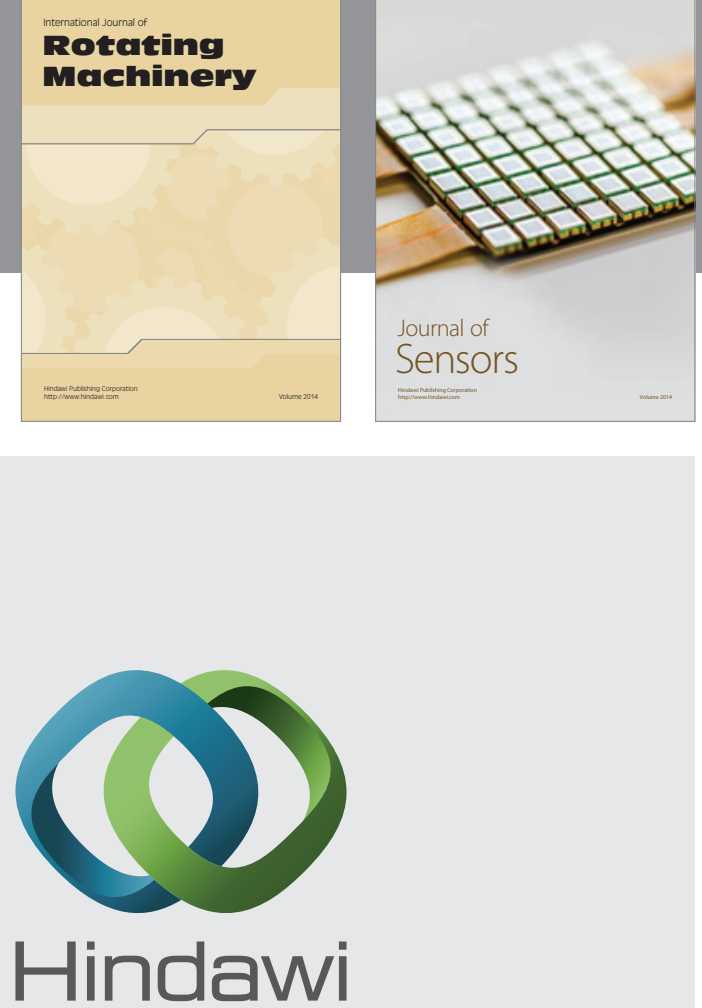

Submit your manuscripts at http://www.hindawi.com
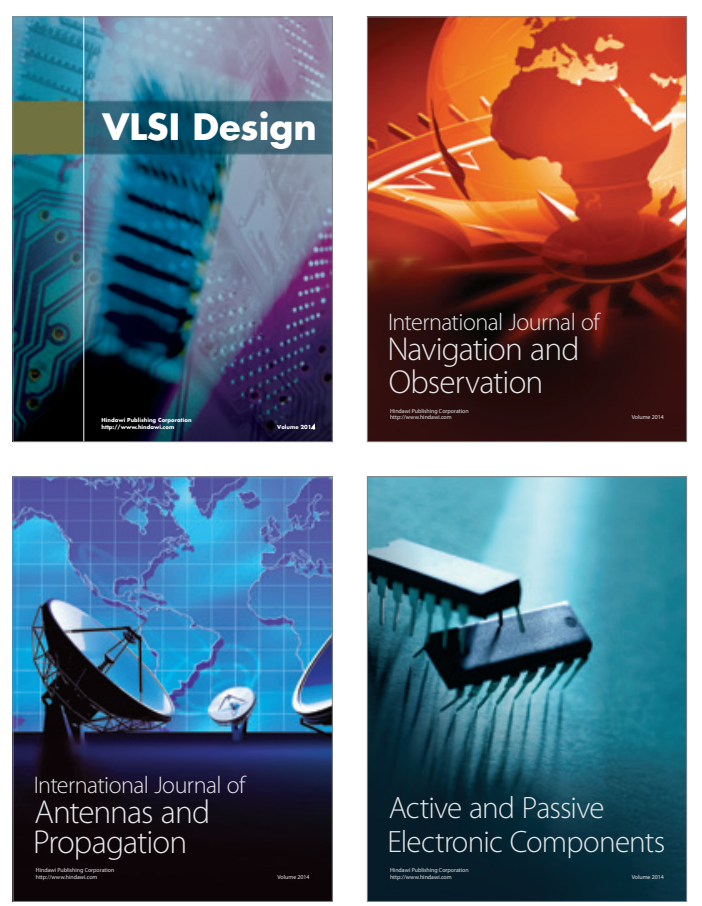
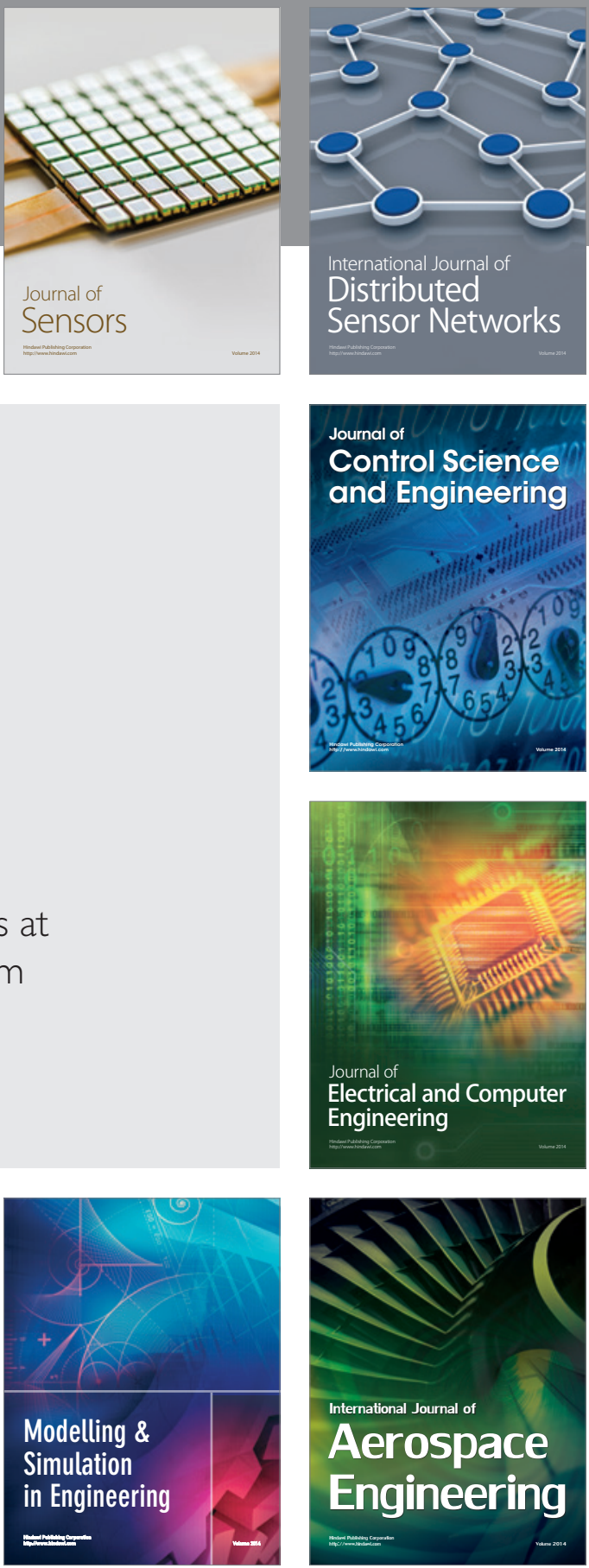

Journal of

Control Science

and Engineering
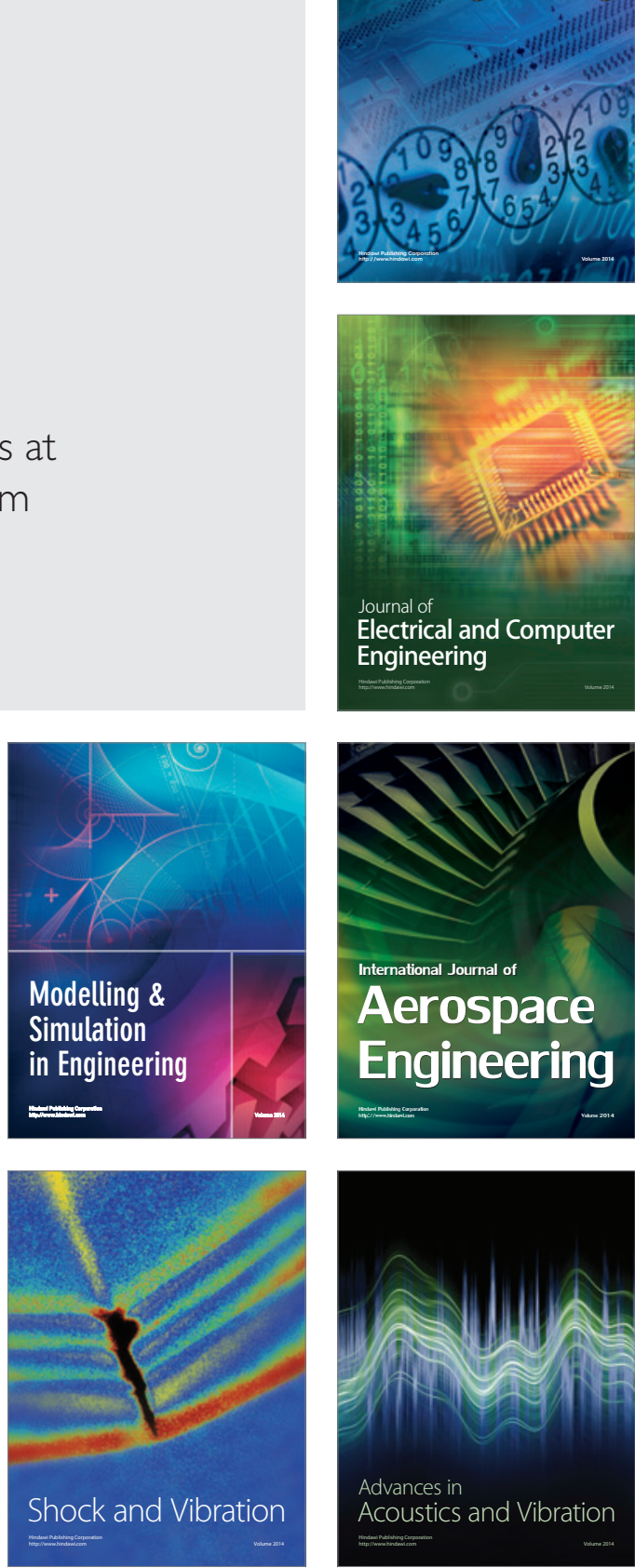